\title{
Asociación de floraciones de algas nocivas y Vibrio spp. en áreas de pesca y acuicultura de bivalvos de moluscos en las bahías de Sechura y Pisco, Perú
}

\section{Harmful algal blooms and Vibrio spp. association in fishing and marine farming areas of mollusk bivalves in Sechura and Pisco bays, Peru}

\section{Rita Orozco ${ }^{1 *}$, Yessica Quispe ${ }^{1}$, Alberto Lorenzo ${ }^{1}$ y María Luz Zamudio ${ }^{2}$}

\author{
1. Instituto del Mar del Perú. Esquina Gamarra y General Valle s/n Chucuito, Callao, Perú. Apartado 22. \\ 2. Instituto Nacional de Salud- Laboratorio de Referencia Nacional de Enteropatogenos, Capac Yupanqui 1400 Jesús María, Lima, Perú. \\ *Autor para correspondencia \\ Email Rita Orozco: ritaoro@imarpe.gob.pe \\ Email Yessica Quispe: yquispe@imarpe.gob.pe \\ Email Alberto Lorenzo: alorenzo@imarpe.gob.pe \\ Email María Luz Zamudio: mzamudio@ins.gob.pe
}

\begin{abstract}
Resumen
Entre febrero de 2010 y mayo de 2014 se realizaron 22 evaluaciones en Pisco y 16 en Sechura; ambas áreas importantes en Perú para la extracción de moluscos y maricultura. La incidencia de Vibrio en el agua de mar fue monitoreada durante las floraciones de algas y en épocas normales. Se midieron parámetros ambientales tales como temperatura y nutrientes. En Sechura, Pseudo-nitzschia seriata y Protoperidinium depressum causaron floraciones de algas y fueron dominantes durante todo el período de evaluación. Las temperaturas en esta zona oscilaron entre 21.8 y $25.3^{\circ} \mathrm{C}$. En Pisco, la concentración Akashiwo sanguinea, Messodinium rubrum y Prorocentrum mínimum y el dinoflagelado Cochlodinium polikrykoides fueron los más frecuentes. Las floraciones de algas nocivas ocurrieron cuando las temperaturas estaban entre 17.1 y $23.3^{\circ} \mathrm{C}$, concentraciones de fosfatos que oscilaban entre $1.22-6.85 \mu \mathrm{M}$ y nitratos $0.15-7.85 \mu \mathrm{M}$. En mayo de 2012, el dinoflagelado Alexandrium peruvianum causó una floración algal, bajo temperaturas de 18.0 a $23.2{ }^{\circ} \mathrm{C}$, valores de fosfato de 0.73 a $11.56 \mu \mathrm{M}$ y nitratos de 0.76 a $9.81 \mu \mathrm{M}$. Los coliformes fueron bajos $<2-23 \mathrm{MPN} / 100 \mathrm{~mL}$, en ambas bahías durante todo el período de estudio. Vibrio alginolyticus fue la especie dominante. Mientras que V. vulnificus y V. parahaemolyticus se detectaron en Pisco, donde las temperaturas del mar comúnmente son mayores y los casos de infecciones graves por ingestión de mariscos se han asociado con el patógeno V. parahaemolyticus.
\end{abstract}

Palabras clave: temperatura; nutrientes; Vibrio; floraciones de algas; moluscos bivalvos; maricultura

\section{Abstract}

Between February 2010 and May 2014, 22 surveys in Pisco and 16 in Sechura were conducted; both are major areas for shellfish production and mariculture in Peru. The incidence of Vibrio in seawater was monitored during algal blooms and in their absence. Environmental parameters such as temperature and nutrients were measured. In Sechura, Pseudo-nitzschia seriata and Protoperidinium depressum caused algal blooms and were dominant throughout the evaluation period. The temperatures in this area ranged from 21.8 to $25.3^{\circ} \mathrm{C}$. In Pisco, the harmful algal bloom-forming Akashiwo sanguinea, Messodinium rubrum, and Prorocentrum minimum and the dinoflagellate Cochlodinium polikrykoides were most prevalent. Harmful algal blooms occurred when temperatures were between 17.1 and $23.3^{\circ} \mathrm{C}$, with phosphates ranging $1.22-6.85 \mu \mathrm{M}$ and nitrates $0.15-7.85 \mu \mathrm{M}$. In May 2012, the dinoflagellate Alexandrium peruvianum caused an algal bloom, with temperatures ranging 18.0 to $23.2^{\circ} \mathrm{C}$, phosphate values from 0.73 to $11.56 \mu \mathrm{M}$, and nitrates from 0.76 to $9.81 \mu \mathrm{M}$. Coliforms were low, $<2-23 \mathrm{MPN} / 100 \mathrm{ml}$, in both bays throughout the study period. Vibrio alginolyticus was the dominant Vibrio spp. predominated in both bays, while $V$. vulnificus and $V$. parahaemolyticus were detected in Pisco, where warmer sea temperatures are common and severe infections cases by seafood ingestion has been associated with a pathogen $V$. parahaemolyticus.

Keywords: temperature; nutrients; Vibrio; algal blooms; bivalve mollusks; mariculture

Citación:

Orozco R., Y. Quispe, A. Lorenzo \& M. Zamudio. 2017. Asociación de floraciones de algas nocivas y Vibrio spp. en áreas de pesca y acuicultura de bivalvos de moluscos en las bahías de Sechura y Pisco, Perú. Revista peruana de biología 24(1): 111 - 116 (Abril 2017). doi: http:// dx.doi.org/10.15381/rpb.v24i1.13111

\section{Presentado: $\quad 26 / 06 / 2016$}

Aceptado: $\quad 12 / 02 / 2017$

Publicado online: $20 / 03 / 2017$
Información sobre los autores:

$\mathrm{RO}$ realizo la concepción del trabajo, el diseño de muestreo, análisis microbiológico y los análisis de la información. YQ realizo análisis de fitoplancton. AL realizo el análisis de nutrientes y de los parámetros fisicoquímicos. MZ realizo los análisis moleculares para determinar factores de virulencia.

Los autores no incurren en conflictos de intereses.

Fuentes de financiamiento: El presente trabajo se realizó con el apoyo del Programa de científico de IMARPE de Evaluación la Calidad del Ambiente marino costero y aguas continentales en áreas seleccionadas, a través de una red de monitoreo.

Journal home page: http://revistasinvestigacion.unmsm.edu.pe/index.php/rpb/index

(c) Los autores. Este artículo es publicado por la Revista Peruana de Biología de la Facultad de Ciencias Biológicas, Universidad Nacional Mayor de San Marcos. Este es un artículo de acceso abierto, distribuido bajo los términos de la Licencia Creative Commons Atribución-NoComercial-Compartirlgual 4.0 Internacional.(http://creativecommons.org/licenses/by-nc-sa/4.0/), que permite el uso no comercial, distribución y reproducción en cualquier medio, siempre que la obra original sea debidamente citadas. Para uso comercial, por favor póngase en contacto con editor.revperubiol@gmail.com. 


\section{Introducción}

Las mareas rojas son fenómenos naturales que se producen como resultado de interacciones físicas, químicas y biológicas. Se han señalado las interacciones como la combinación de vientos, surgencias, frentes de densidad, concentraciones de nutrientes o alteraciones antropogénicas (Alonso \& Ochoa 2004, Buschmann 2005, Pitcher et al. 1998, Ryan et al. 2009, Kudela et al. 2005). La intensidad y distribución geográfica de las floraciones algales causadas por dinoflagelados y diatomeas han aumentado en las últimas décadas, asociadas a cambios climáticos locales o globales (Wells et al. 2015, Klais et al. 2011, Hinder et al 2012, Edwards et al. 2006, Reguera 2002, Hallegraeff 1993); así como también el incremento de los reportes de casos de intoxicación humana y graves pérdidas económicas en la industria pesquera (Brooks et al. 2016, Sanseverino et al. 2016, Berdalet et al. 2015, Ferrante et al. 2013, Anderson et al. 2002, Anderson et al. 2000). Además, el estrés antropogénico produce cambios que aumentan la diversidad de comunidades bacterianas marinas y la variabilidad temporal (Davidson et al. 2014, Anderson et al. 2008, Nogales et al. 2011).

Durante las floraciones de algas también es común encontrar bacterias en el género Vibrio, reportándose posibles interacciones entre las algas y las bacterias (Seong \& Jeong 2011), por ejemplo se mencionana efectos alguicidas de las bacterias sobre dinoflagelados (Li et al. 2015, Wang et al. 2010) y otras interacciones comunitarias (Thomas et al. 2014). Esto indica que las floraciones de algas pueden tener consecuencias adversas incluyendo efectos sobre la dinámica de comunidades de bacterias como las especies de Vibrio (Imai et al. 2006). Además, los vibrios están ampliamente distribuidos en aguas costeras e incluyen especies patógenas y no patógenas (Eiler et al. 2006).

La presencia de Vibrio spp. y su posible asociación con las floraciones de algas puede ser una amenaza para la maricultura y las actividades pesqueras, así como también para la salud humana. Por estos motivos, en este trabajo se determinó la presencia de Vibrio en el agua de mar durante eventos de floración de algas y en períodos normales, en dos de las áreas de cultivo más importantes para los moluscos bivalvos (bahías Sechura y Pisco) en Perú, para investigar la posible relación entre estas especies.

\section{Material y métodos}

La bahía de Sechura está situada entre los 518'46” - 5 50'33"S (Fig. 1a) y la Bahía de Pisco entre 13³9'35.1”-1349'19.6”S (Fig. 1b). Las muestras de agua y plancton fueron recolectadas en frascos estériles durante las floraciones algales nocivas y no nocivas sucedidas entre 2010 - 2014. Inmediatamente después del muestreo, las muestras de agua para análisis microbiológicos se almacenaron en un recipiente aislado a $4{ }^{\circ} \mathrm{C}$. Las muestras de plancton se conservaron en formalina neutralizada, según lo recomendado por Throndsen (1978). La determinación taxonómica del fitoplancton se realizó mediante procedimientos de Hustedt (1930), Cupp (1943), Hendey (1964), Sournia (1967) Schiller (1971), Sundström (1986), Heimdal (1993) y Steidinger y Tangen (1996) según lo recomendado por Delgado et al 2015.

El aislamiento de Vibrio se realizó con un enriquecimiento selectivo de las muestras, sembrando volúmenes iguales de Agua Peptonada Alcalina (APA) 2X (pH 8.5) y agua de mar y se incubo a $30^{\circ} \mathrm{C}$ por $18-24$ horas. Posteriormente se sembró por el método de estriado en placas con Agar TCBS (tiosulfato, citrato, sales biliares, sacarosa), luego se seleccionó de cada placa una colonia sacarosa positiva y/o sacarosa negativas de manera que se asegure la pureza de las cepas características de las especies de Vibrio. Se realizó la identificación bioquímica tradicional según Llop et al. (2001) (sacarosa, oxidasa, Lisina, ornitina, lactosa, indol, manitol, Cloruro de Sodio al 0, 1, 6, 7 y 10\%). La identificación utilizando el sistema $\mathrm{API}^{\odot} 20$ NE Microbial Identification Kit, siguiendo el procedimiento indicado por el fabricante Biomérieux S. A. 2009/11 y el software API WEB.

En las cepas Vibrio parahaemolyticus aisladas de agua de mar, para estudiar los factores de virulencia se utilizó el protocolo de PCR para detectar el gen toxR (Kim et al., 1999) y para la detección de los genes de tdh y trh (Tada et al., 1992).
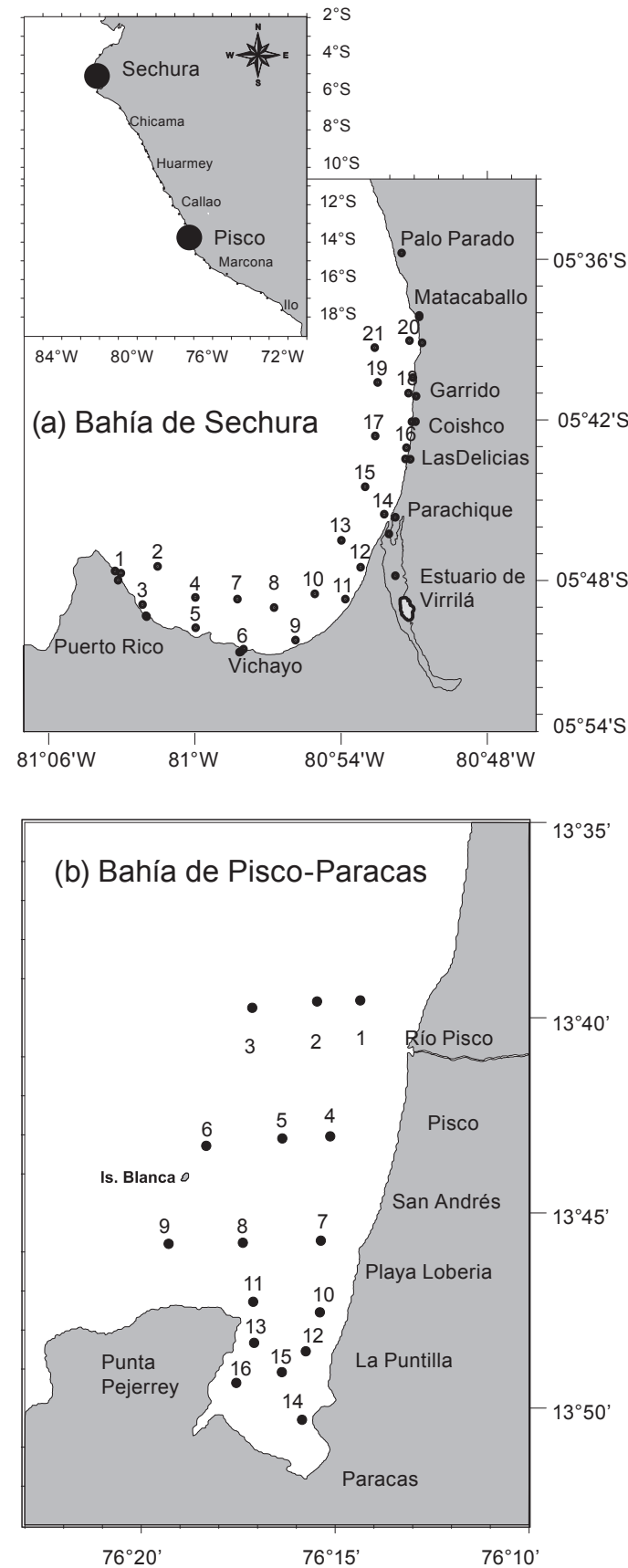

Figura 1. Área de estudio y ubicación de los sitios de muestreo en las bahías de Sechura y Pisco, Perú. 


\section{Resultados}

Parámetros Fisicoquímicos.- Las mareas rojas en la costa peruana son muy frecuentes en primavera y verano, coincidiendo con la intensidad de afloramiento. Este proceso se caracteriza por el movimiento a la superficie de aguas frías ricas en nutrientes, con salinidades relativamente bajas menores de 35.0 ups, donde las algas microscópicas y otros fitoplancton crecen abundantemente (Zuta \& Guillen 1970, Graco et al. 2007).

En el período evaluado, las características fisicoquímicas de la bahía de Sechura fueron similares a la bahía de Pisco (temperatura, salinidad y pH) (Tabla 1). Sin embargo, resalta la elevada variabilidad en la concentración de oxígeno disuelto que se observó en la bahía de Pisco, con registros de valores saturados relacionados a floraciones algales y los valores hipóxicos cercanos a la anoxia $\left(0.07 \mathrm{mg} \mathrm{O}_{2} / \mathrm{L}\right)$, que se observan en períodos posteriores estas floraciones.

Las especies dominantes en las floraciones de algales (mareas rojas) fueron:

En Sechura, Grupo Pseudo-nitzschia seriata y el dinoflagelado Protoperidinium depressum, en temperaturas entre 21.8 y $25.3^{\circ} \mathrm{C}$.

En Pisco, Akashiwo sanguinea, el ciliado Messodinium rubrum, y el dinoflagelado tecado Prorocentrum minimun, fueron los dominantes de mayor frecuencia; sin embargo destaca Cochlodinium polykrikoides dominante entre 17.1 y $23.3^{\circ} \mathrm{C}$. En mayo de 2012, Alexandrium peruvianum fue la causa de una floración algal cuando las temperaturas estuvieron entre 18.0 y $23.2^{\circ} \mathrm{C}$. En abril y mayo de 2012 hubo mortalidad de moluscos bivalvos debido a condiciones anóxicas en aguas de fondo, con presencia de una floración de Alexandrium peruvianum.

Las especies de Vibrio patógenas detectadas durante las prospecciones fueron Vibrio parahaemolyticus, V. vulnificus, $V$. mimicus, $V$. alginolyticus y $V$. hollisae. Se han reportado a Vibrio parahaemolyticus como el principal agente causal de la gastroenteritis aguda humana transmitida a través del consumo de mariscos crudos o poco cocidos (Ibarra et al 2007, Gil et al., 2007).

Las especies de Vibrio que dominaron en frecuencia en las bahías fueron:

- Vibrio alginolitycus (62\%), V. parahaemolyticus (17\%), $V$. mimicus (5\%) y $V$. metschnikovii (3\%) en la bahía de Sechura (Fig. 2a)

- Vibrio alginolyticus (84\%), V. parahaemolyticus (8\%) y $V$. metschnikovii (8\%) los más prevalentes en la bahía de Pisco (Fig. 2b)
Durante el periodo de estudio, las cinco especies de Vibrio identificadas se asociaron a la presencia de 20 especies de plancton identificadas como causantes de mareas rojas en la Bahía de Sechura y Pisco y se observándose 38 combinaciones. Las combinaciones más comunes en estas bahías fueron: $V$. alginolyticus - Messodinium rubrum (10.1\% de todos los eventos), $V$. alginolyticus-Grupo Pseudo-nitzschia seriata (6.1\%), V. alginolyticus-Licmophora abbreviata (6.1\%), Alexandrium peruvianum - V. alginolyticus - Heterosigma akashiwo y V. parahaemolyticus - Heterosigma akashiwo (3.0\%) (Tabla 2)

La incidencia de Vibrio parahaemolyticus es alta en aguas costeras y cultivos marinos; su presencia en el ambiente marino ha sido demostrada en varios estudios (Lopez-Joven et al. 2015, Aliaga et al. 2011, Gil et al. 2007, DePaola et al. 1990). En el Perú también se viene realizando la vigilancia epidemiológica de esta bacteria y otros patógenos con técnicas moleculares en el Instituto Nacional de Salud y algunos estudios en aguas marinas. Por esta razón de las 138 cepas de Vibrio sp. que fueron aisladas de aguas marinas de las bahías de Sechura y Pisco durante la ocurrencia de floraciones algales, solo 16 cepas fueron confirmadas para V.parahaemolyticus

(a) Sechura $2010-2014$

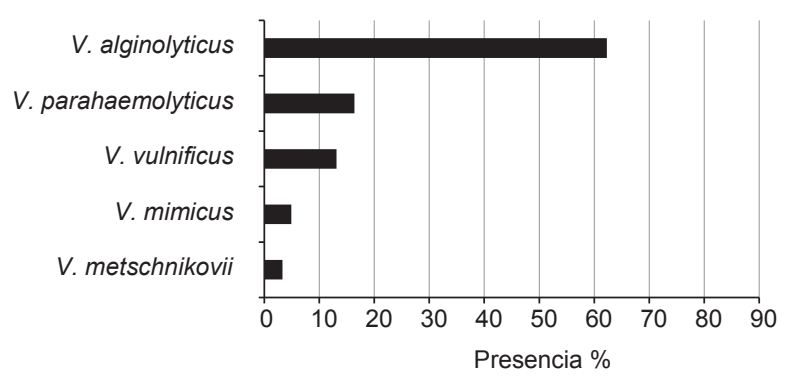

(b)

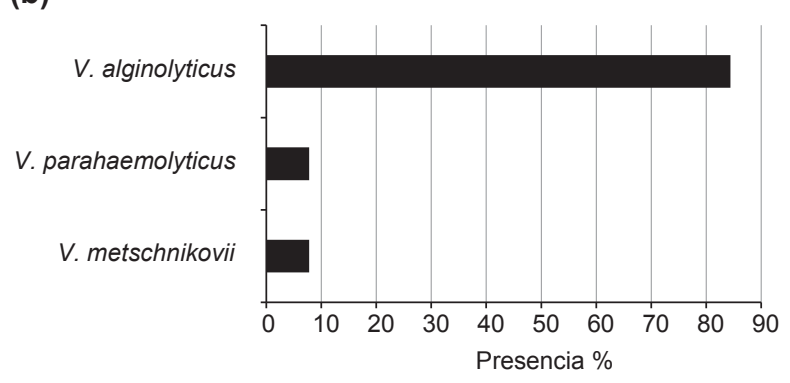

Figura 2. Presencia de Vibrio sp. en las bahías de Sechura y Pisco, Perú.

Tabla 1. Parámetros físico-químicos en las bahías de Sechura y Pisco, Perú 2010 - 2014.

\begin{tabular}{|c|c|c|c|c|c|c|}
\hline Area & $\mathbf{n}$ & Valor & $\operatorname{TSM}\left({ }^{\circ} \mathrm{C}\right)$ & Salinidad (psu) & Oxygeno (mg/L) & $\mathrm{pH}$ \\
\hline \multirow{3}{*}{ Sechura } & \multirow{3}{*}{116} & Max. & 23.9 & 35.34 & 8.21 & 8.19 \\
\hline & & Min. & 14.7 & 34.8 & 2.76 & 6.87 \\
\hline & & Ave. & 19.02 & 34.94 & 5.04 & 7.83 \\
\hline \multirow{3}{*}{ Pisco } & \multirow{3}{*}{113} & Max. & 24.8 & 35.22 & 13.21 & 8.5 \\
\hline & & Min. & 13.9 & 32.71 & 0.07 & 6.92 \\
\hline & & Ave. & 17.66 & 34.86 & 5.31 & 7.78 \\
\hline
\end{tabular}

$\mathrm{n}=$ number of record per site 
Tabla 2. Combinación de cepas de Vibrio sp y especies de Floraciones Algales aisladas de los períodos 2010-2014

\begin{tabular}{|c|c|c|c|c|c|c|}
\hline & Vibrio sp. & HABs & Sechura & Pisco & $\%$ & \# Site \\
\hline 1 & V. alginolyticus & Alexandrium peruvianum & 0 & 5 & 5.1 & 5 \\
\hline 2 & V.mimicus & Alexandrium peruvianum & 0 & 1 & 1.0 & 1 \\
\hline 3 & V. alginolyticus & Chaetoceros curvisetus & 5 & 0 & 5.1 & 5 \\
\hline 4 & V. alginolyticus & Chaetoceros debilis & 0 & 2 & 2.0 & 1 \\
\hline 5 & V. vulnificus & Chaetoceros debilis & 0 & 1 & 1.0 & 1 \\
\hline 6 & V. alginolyticus & Chaetoceros socialis & 4 & 0 & 4.0 & 21 \\
\hline 7 & V. parahaemolyticus & Chaetoceros socialis & 3 & 0 & 3.0 & 4 \\
\hline 8 & V. alginolyticus & Cochlodinium polykrikoides & 0 & 4 & 4.0 & 3 \\
\hline 9 & V. metschnikovii & Cochlodinium polykrikoides & 0 & 1 & 1.0 & 1 \\
\hline 10 & V. vulnificus & Cochlodinium polykrikoides & 0 & 2 & 2.0 & 2 \\
\hline 11 & V. alginolyticus & Coscinudiscus granii & 3 & 0 & 3.0 & 3 \\
\hline 12 & V. alginolyticus & Dinophysis acuminata & 0 & 2 & 2.0 & 2 \\
\hline 13 & $V$. mimicus & Dinophysis acuminata & 0 & 1 & 1.0 & 1 \\
\hline 14 & V. alginolyticus & Dinophysis caudata & 0 & 1 & 1.0 & 1 \\
\hline 15 & V. alginolyticus & Dinophysis rotundata & 2 & 0 & 2.0 & 2 \\
\hline 16 & V. alginolyticus & Dinophysis rotundata & 2 & 0 & 2.0 & 2 \\
\hline 17 & V. metschnikovii & Dinophysis rotundata & 2 & 0 & 2.0 & 1 \\
\hline 18 & V. alginolyticus & Dinophysis sp & 4 & 0 & 4.0 & 3 \\
\hline 19 & V. metschnikovii & Dinophysis sp & 2 & 0 & 2.0 & 2 \\
\hline 20 & V. vulnificus & Gonyaulax Polygramma & 0 & 3 & 3.0 & 3 \\
\hline 21 & V. parahaemolyticus & Heterosigma akashiwo & 0 & 3 & 3.0 & 1 \\
\hline 22 & V. alginolyticus & Licmophora lingbyei & 6 & 0 & 6.1 & 6 \\
\hline 23 & V. alginolyticus & Messodinium rubrum & 10 & 0 & 10.1 & 10 \\
\hline 24 & V. parahaemolyticus & Messodinium rubrum & 0 & 3 & 3.0 & 1 \\
\hline 25 & V. alginolyticus & Prorocentrum minimum & 0 & 3 & 3.0 & 2 \\
\hline 26 & V. vulnificus & Prorocentrum minimum & 0 & 1 & 1.0 & 1 \\
\hline 27 & V. alginolyticus & Protoperidinium depressum & 0 & 1 & 1.0 & 1 \\
\hline 28 & V. alginolyticus & Protoperidinium depressum & 1 & 0 & 1.0 & 1 \\
\hline 29 & V. alginolyticus & Pseudo-nitzschia pungens & 6 & 0 & 6.1 & 3 \\
\hline 30 & V. metschnikovii & Pseudo-nitzschia pungens & 2 & 0 & 2.0 & 2 \\
\hline 31 & V. parahaemolyticus & Pseudo-nitzschia pungens & 0 & 1 & 1.0 & 1 \\
\hline 32 & $V$. parahaemolyticus & Pseudo-nitzschia pungens & 2 & 0 & 2.0 & 2 \\
\hline 33 & V. alginolyticus & Pseudo-nitzschia seriata & 4 & 0 & 4.0 & 4 \\
\hline 34 & V. alginolyticus & Pseudo-nitzschia. cf. delicatissima & 0 & 2 & 2.0 & 2 \\
\hline 35 & V. alginolyticus & Pseudo-nitzschia. cf. delicatissima & 1 & 0 & 1.0 & 1 \\
\hline 36 & V. metschnikovii & Pseudo-nitzschia. cf. delicatissima & 0 & 1 & 1.0 & 1 \\
\hline 37 & V. parahaemolyticus & Pseudo-nitzschia. cf. delicatissima & 0 & 1 & 1.0 & 1 \\
\hline 38 & V. mimicus & V. mimicus- Dinophysis caudata & 0 & 1 & 1.0 & 1 \\
\hline
\end{tabular}

las que fueron sometidas a exámenes moleculares para la detección del gen toxR y los genes (tdh + , toxR y trh-). Los resultados indican que en un aislamiento recuperado de V.parahaemolyticus en febrero de 2011 durante una floración algal producida por Prorocentrun minimum en la bahía de Pisco dio positivo para estos factores de virulencia, asociado a una TSM de $20.1^{\circ} \mathrm{C}$.

\section{Conclusiones}

En el periodo de estudio del 2010 al 2014, en las bahías de Sechura y Pisco predominó en número Vibrio alginolyticus, seguido de V. parahaemolyticus en agua de mar. Se ha demostrado la coexistencia entre la presencia de estos Vibrio con las especies de diatomeas y dinoflagelados productores de floraciones algales nocivas.
La detección de factores en virulencia (genes $t d h$ and $t r h$ ) en Vibrio parahaemolyticus aislado en Sechura y Pisco durante durante el periodo evaluado del 2010 y 2014, solo dio positivo para una cepa aislada en Pisco, en febrero del 2011 y asociado a la temperatura de la estación de verano.

Estos resultados señalan la necesidad de realizar un monitoreo continuo de estas especies para manejar sus efectos sobre la calidad acuática y recursos y prevenir los efectos adversos sobre la salud humana.

\section{Agradecimiento}

Agradecemos al personal del Laboratorio de Microbiología Acuática, Oceanografía de IMARPE - Paita y Pisco. También a la Licenciada Tecnólogo Medico Carmela Aguilera Rodríguez 
del Instituto Nacional de Salud por el ensayo de PCR colaboración en esta investigación. Un agradecimiento especial al Dr. Angelo DePaola por sus valiosas sugerencias y recomendaciones a este trabajo.

\section{Literatura citada}

Aliaga R., J. Miranda \& J. Zevallos. (2011). Aislamiento e identificación de Vibrio parahaemolyticus O3:K6 en pescados y moluscos bivalvos procedentes de un mercado pesquero de Lima, Perú. Revista Médica Herediana 21 (3):139-145

Alonso R.R. \& J.L. Ochoa. 2004. Hydrology of winter-spring "red tides" in Bahía de Mazatlán, Sinaloa, México. Harmful Algae 3, 163-171. http://doi.org/10.1016/j.hal.2003.10.002

Anderson D.M., P. Hoagland, Y. Kaoru, A.W. White. 2000. Estimated Annual Economic Impacts from Harmful Algal Blooms (HABs) in the United States. Technical Report WHOI2000-11, Woods Hole Oceanographic Institution.

Anderson D.M., P.M. Glibert \& J.M. Burkholder. 2002. Harmful Algal Blooms and Eutrophication: Nutrient Sources, Composition, and Consequences. Estuaries 25 (4): 704-26. http:// doi.org/10.1007/BF02804901.

Anderson D.M., J.M. Burkholder, W.P. Cochlan, P.M. Glibert, C.J. Gobler, C.A. Heil, R.M. Kudela, et al. 2008. Harmful algal blooms and eutrophication: Examining linkages from selected coastal regions of the United States. Harmful Algae, HABs and Eutrophication, 8 (1): 39-53. http://doi. org/10.1016/j.hal.2008.08.017.

Berdalet E., L.E. Fleming, R. Gowen, K. Davidson, P. Hess, L.C. Backer, S.K. Moore, P. Hoagland \& H. Enevoldsen. 2015. Marine harmful algal blooms, human health and wellbeing: challenges and opportunities in the 21 st century. Journal of the Marine Biological Association of the United Kingdom. http://www.ncbi.nlm.nih.gov/pmc/articles/PMC4676275/.

Buschmann A. 2005. Marea roja y salmonicultura en el Sur de Chile. Documento 14. Chile: Fundación Oceana. Disponible en: $<$ www.oceana.org $>$.

Brooks B.W., J.M. Lazorchak, M.D. A. Howard, M.V.V. Johnson, S.L. Morton, D.A.K. Perkins, E.D. Reavie, G.I. Scott, S.A. Smith \& J.A. Steevens. 2016. Are Harmful Algal Blooms Becoming the Greatest Inland Water Quality Threat to Public Health and Aquatic Ecosystems? Environmental Toxicology and Chemistry 35 (1): 6-13. http://doi.org/10.1002/etc.3220.

Cupp E. 1943. Marine plankton diatoms of the west coast of North America. Bull. Scripps Inst. Oceangr. 5: 1-237. http:// escholarship.org/uc/item/922945w8

Davidson K., R.J. Gowen, P.J. Harrison, L.E. Fleming, P. Hoagland \& G. Moschonas. 2014. Anthropogenic nutrients and harmful algae in coastal waters. Journal of Environmental Management 146 (diciembre): 206-16. http://doi.org/10.1016/j. jenvman.2014.07.002.

DePaola A., L.H. Hopkins, J.T. Peeler, B. Wentz, y R.M. McPhearson. (1990). Incidence of Vibrio parahaemolyticus in U.S. coastal waters and oysters. Applied and Environmental Microbiology 56 (8): 2299-2302.

Delgado E., F. Chang, A. Bernales 2015. Fitoplancton del mar peruano en el verano 2008. Informe Instituto del Mar del Perú 42(2):172-177

Edwards M., D.G. Johns, S.C. Leterme, E. Svendsen \& A.J. Richardson. 2006. Regional Climate Change and Harmful Algal Blooms in the Northeast Atlantic. Limnology and Oceanography 51 (2): 820-29. doi:10.4319/lo.2006.51.2.0820.

Eiler A.; M. Johansson \& S. Bertilsson. 2006. Environmental influences on Vibrio populations in northern temperate and boreal coastal waters (Baltic and Skagerrak Seas). Applied and Environmental Microbiology 72 (9): 6004-6011.

Ferrante M., S. Sciacca, R. Fallico, M. Fiore, G.O. Conti, et al. 2013. Harmful Algal Blooms in the Mediterranean Sea: Effects on Human Health. 2:587 doi:10.4172/scientificreports.587

Gil, A.; Lanata, C.; Miranda, H.; Prada, A.; Seas, C.; Hall, E.; Meza, R.; Barreno, C.; Maurtua, D. y Nair, B. (2007). Gravedad de la gastroenteritis causada por Vibrio parahaemolyticus del grupo pandémico en el Perú. Revista Peruana de Medicina Experimental y Salud Publica 24 (4): 350-355.
Graco M.I., J. Ledesma, G. Flores \& M. Girón. (2007). Nutrientes, oxígeno y procesos biogeoquímicos en el sistema de surgencias de la corriente de Humboldt frente a Perú. Revista peruana de biología 14(1):117-128 http://dx.doi. org/10.15381/rpb.v14i1.2165

Hallegraeff G.M .1993. A review of harmful algal blooms and their apparent global increase. Phycologia 32: 79-99. http:// dx.doi.org/10.2216/i0031-8884-32-2-79.1

Heimdal B. 1993. Modern Coccolithophorids. In Tomas C. (ed.). Marine Phytoplankton a guide to naked flagellates and coccolithophorids, Cap. 3. Academic Press, Inc. San Diego. 147-235 pp.

Hendey I. 1964. An introductory account of the smaller algae of British Coastal waters. Part. V. Bacillariophyceae (Diatoms). Her Majesty's Stationery Office, London: 317 pp.

Hinder S.L., G.C. Hays, M. Edwards, E.C. Roberts, A.W. Walne \& M.B. Gravenor. 2012. Changes in marine dinoflagellate and diatom abundance under climate change. Nature Climate Change 2 (4): 271-75 . http://dx.doi.org/10.1038/ nclimate 1388

Hustedt F. 1930. Die Kieselalgen Deutschlands. Osterreichs und der Schqeiz mit Beucksichtigung der ubrigen Lander Europas sowie der angerzenden Meerresgebiete. En: L. Rabenhorst (ed). Kryptogamen-Flora von Deutschland, Osterreich und der Schweiz. 1 Teil. Akat. Verlagsges. Leipzig, Reprint Johnson Rep. Goop, New York 1971, 920 pp.

Ibarra J.O. \& D.E. Alvarado. 2007. Antimicrobial resistance of clini$\mathrm{cal}$ and environmental strains of Vibrio cholerae isolated in Lima-Peru during epidemics of 1991 and 1998. Brazilian Journal of Infectious Diseases 11(1), 100-105. http://dx.doi. org/10.1590/S1413-86702007000100022

Imai I., M. Yamaguchi \& Y. Hori. (2006). Eutrophication and occurrences of harmful algal blooms in the Seto Inland Sea, Japan. Plankton and Benthos Research 1 (2): 71-84. http://dx.doi. org/10.3800/pbr.1.71.

Kim Y.B., J. Okuda, C. Matsumoto, N. Takahashi, S. Hashimoto, M. Nishibuchi. 1999. Identification of Vibrio parahaemolyticus at the species level by PCR targeted to the toxR gene. Journal of Clinical Microbiology37:1173-1177

Klais R., T. Tamminen, A. Kremp, K. Spilling, \& K. Olli. 2011. Decadal-Scale Changes of Dinoflagellates and Diatoms in the Anomalous Baltic Sea Spring Bloom. PLoS ONE 6 (6). http://dx.doi.org/10.1371/journal.pone.0021567.

Kudela R., G. Pitcher, T. Probyn, F. Figueiras, T. Moita, and V. Trainer. 2005. Harmful algal blooms in coastal upwelling systems. Oceanography 18(2):184-197, http://dx.doi.org/10.5670/ oceanog.2005.53.

Li Y., H. Zhu, X. Lei, H. Zhang, G. Cai, Z. Chen, L. Fu, H. Xu \& T. Zheng. 2015. The Death Mechanism of the Harmful Algal Bloom Species Alexandrium Tamarense Induced by Algicidal Bacterium Deinococcus Sp. Y35. Frontiers in Microbiology 6. http://dx.doi.org/10.3389/fmicb.2015.00992.

Llop A., M. Valdés-Dapena \& J. Zuazo. 2004. Microbiología y parasitología médicas, t. I. La Habana: Editorial Ciencias Médicas

Lopez-Joven C., I. de Blas, M.D. Furones y A. Roque. 2015. Prevalences of pathogenic and non-pathogenic Vibrio parahaemolyticus in mollusks from the Spanish Mediterranean Coast. Food Microbiology, 736. http://dx.doi.org/10.3389/ fmicb.2015.00736.

Nogales B., M.P. Lanfranconi, J.M. Piña-Villalonga \& R. Bosch. 2011. Anthropogenic Perturbations in Marine Microbial Communities. FEMS Microbiology Reviews 35 (2): 27598. http://dx.doi.org/10.1111/j.1574-6976.2010.00248.x.

Pitcher G.C., A.J. Boyd, D.A. Horstman \& B.A. MitchellInnes. 1998. Subsurface dinoflagellate populations, frontal blooms and the formation of red tide in the southern Benguela upwelling system. Marine Ecology Progress Series 172: 253-64. http:// dx.doi.org/10.3354/meps172253.

Reguera B. 2002. Establecimiento de un programa de seguimiento de microalgas toxicas. Floraciones algales nocivas en el cono Suramericano. En: Sar E.A., M.E. Ferrario \& B. Reguera (Eds.). Madrid. Instituto Español de Oceanografía. pp: 5-54

Sanseverino I., D. Conduto, L. Pozzoli, S. Dobricic \& T. Lettieri. 2016. Algal bloom and its economic impact; EUR 27905 EN; http://dx.doi.org/10.2788/660478 
Seong K.A. \& H.J. Jeong. 2011. Interactions between the Pathogenic Bacterium Vibrio Parahaemolyticus and Red-Tide Dinoflagellates. Ocean Science Journal 46 (2): 105-15. http:// dx.doi.org/10.1007/s12601-011-0010-2.

Schiller J. 1971. Dinoflagellate (peridinae) in monographischer Behandlung.2 Tell. En: L. Rabenhortst (ed). Kriptogamen-Flora von Deutchland, Osterreich und der Schweiz. Reprint by Johnson Repr. Corp. New York, 1971, Vol. 10 Section 3, Parte 1: $617 \mathrm{pp}$.

Steidinger K., Tangen K. 1996. Dinoflagellates. In: Tomas C. (ed.). Identifying Marine Diatoms and Dinoflagellates. Academic Press, Inc. San Diego. 387-570pp.

Sournia A. 1967. Le genre Ceratium (Peridinien Planctonique) dans le Canal de Mozambique. Contribution a une révision mondiale. Vie et Milieu. 18 (2A-A): 375-580 pp.

Sundström B. 1986. The Marine diatom genus Rhizosolenia. A new approach to the taxonomy. Lund, Sweden: $196 \mathrm{pp}$.

Ryan J.P., A.M. Fischer, R.M. Kudela, J.F. R. Gower, S.A. King, R.Marin III \& F.P. Chavez. 2009. Influences of upwelling and downwelling winds on red tide bloom dynamics in Monterey Bay, California. Continental Shelf Research 29 (5-6): 785-95. http://dx.doi.org/10.1016/j.csr.2008.11.006.
Tada J., T. Ohashi, N. Nishimura, Y. Shirasaki, H. Ozaki, S. Fukushima, J. Takano, M. Nishibuchi \& Y. Takeda. 1992. Detection of the thermostable direct hemolysin gene (tdh) and the thermostable direct hemolysin-related hemolysin gene (trh) of Vibrio parahaemolyticus by polymerase chain reaction. Molecular and Cellular Probes 6 (6): 477-87. http://dx.doi. org/10.1016/0890-8508(92)90044-X.

Thomas A.M., M.G. Sanilkumar, K.C. Vijayalakshmi, A.A. Mohamed Hatha \& A.V. Saramma. 2014. Dynamic Changes in Bacterial Population and Corresponding Exoenzyme Activity in Response to a Tropical Phytoplankton Bloom Chattonella Marina. Journal of Marine Biology: e918614. http://dx.doi. org/10.1155/2014/918614.

Zuta S. \& O.G. Guillén. 1970. Oceanografía de las aguas costeras del Perú. Boletín, Instituto del Mar Perú (2):157-324

Wang B.x., Y.y. Zhou, S.j. Bai, J.q. Su, Y. Tian, T.l. Zheng \& X.r. Yang. 2010. A Novel Marine Bacterium Algicidal to the Toxic Dinoflagellate Alexandrium Tamarense. Letters in Applied Microbiology 51 (5): 552-57. http://dx.doi. org/10.1111/j.1472-765X.2010.02936.x.

Wells M.L., V.L. Trainer, T.J. Smayda, B.S.O. Karlson, C.G. Trick, R.M. Kudela, A. Ishikawa, et al. 2015. Harmful algal blooms and climate change: Learning from the past and present to forecast the future. Harmful algae 49: 68-93. http://dx.doi. org/10.1016/j.hal.2015.07.009. 